Annuaire suisse de politique de développement

Société de l'information et coopération internationale

\title{
Radio, Internet et satellite au Sénégal pour lutter contre les feux de brousse
}

\section{Martin Faye}

\section{(2) OpenEdition}

1 Journals

Édition électronique

URL : http://journals.openedition.org/aspd/552

DOI : 10.4000/aspd.552

ISSN : 1663-9669

Éditeur

Institut de hautes études internationales et du développement

Édition imprimée

Date de publication : 1 novembre 2003

Pagination : 89-91

ISSN : 1660-5934

\section{Référence électronique}

Martin Faye, «Radio, Internet et satellite au Sénégal pour lutter contre les feux de brousse », Annuaire suisse de politique de développement [En ligne], 22-2 | 2003, mis en ligne le 22 mars 2010, consulté le 08 septembre 2020. URL : http://journals.openedition.org/aspd/552 ; DOI : https://doi.org/10.4000/ aspd.552 


\title{
Radio, Internet et satellite au Sénégal pour lutter contre les feux de brousse
}

\author{
Martin Faye*
}

E n Afrique, certains projets utilisent les technologies de l'information et de la communication (TIC) depuis de nombreuses années de manière spontanée. Que la technologie employée soit nouvelle, analogique, vieille ou traditionnelle, qu'importe. Ce qui compte, c'est l'efficacité par rapport aux objectifs visés, la durabilité et l'autonomie. Là où des ordinateurs performants avaient été installés, les groupements paysans ont vite compris que l'achat d'un modem leur permettait de se mettre en réseau avec d'autres groupements situés ailleurs. Ils utilisent, comme beaucoup d'autres, des TIC sans le savoir.

Dans les pays pauvres, c'est sans doute dans le domaine des médias que les TIC ont fait la percée la plus importante. Dans la presse écrite, l'ordinateur a remplacé les anciennes et lourdes technologies de l'imprimerie. A la radio, les anciens équipements ont pratiquement disparu; la bande magnétique n'étant plus fabriquée en masse, elle atteint des prix inaccessibles pour les petites radios. L'audionumérique est en revanche bon marché, mais moins durable et plus exigeant en formation et en maintenance. Bref, tout le secteur de la radio - des radios de proximité aux radios nationales - vit une révolution technologique profonde.
L'ordinateur et le modem ont fait leur entrée dans les studios des radios rurales et sont utilisés de diverses manières, souvent étonnantes: la culture orale africaine est recueillie et conservée sur des supports stables (CD, DVD, disque dur); le courrier électronique s'ouvre aux analphabètes par le truchement des radios rurales, au point de supplanter la poste dans les régions de diaspora, etc. Il en va de même du projet «Pour une meilleure maîtrise des feux de brousse en Afrique de l'Ouest». Soutenu par la DDC, il associe les communautés locales et les services techniques ainsi que le Centre de suivi écologique de Dakar $(\mathrm{CSE})^{1}$, une institution sénégalaise spécialisée dans le suivi satellitaire.

Quoi de plus «vieille technologie» que le feu prométhéen? «Bulldozer du pauvre», les paysans l'utilisent pour le défrichement, la préparation des champs, l'éloignement des serpents des habitations, mais aussi pour la chasse et les rites divinatoires. Pratiqué sur une vaste échelle, le feu est également la seule contribution sérieuse du continent africain au réchauffement global. La pratique des feux précoces, préconisée par la plupart des services techniques d'Afrique de l'Ouest, est cependant acceptable et correspond à des tradi-

\footnotetext{
Directeur d'Intermédia Afrique, Sénégal.

$<$ www.cse.sn>.
} 
tions précoloniales. Ils sont allumés quand l'herbe est encore mouillée, dégagent peu de chaleur et permettent à la nature de se régénérer dès les premières pluies de la nouvelle saison. Les feux tardifs par contre, allumés quand l'herbe est sèche, détruisent les végétaux et les sols.

Les radios rurales sont très efficaces en Afrique, car elles s'inscrivent dans le prolongement de l'oralité. Depuis longtemps elles ont attiré l'attention des communautés locales sur les méfaits des feux de brousse, malheureusement avec des succès très limités, voire même négatifs quand ils deviennent des feux de mécontentement politique. Un dictateur qui les avait interdits était accompagné sur le parcours de ses tournées à l'intérieur du pays par des incendies qui n'avaient rien de feux de joie.

Le travail en réseau par Internet et les images satellites permettent dorénavant une plus grande efficacité. Grâce aux efforts de l'Union européenne notamment, le suivi des feux par satellite en Afrique de l'Ouest au cours des dix dernières années est parfaitement connu. A certaines périodes de l'année, les photos satellites donnent l'impression que toute l'Afrique brûle. Ces données ne parvenaient pas aux communautés de base et les services techniques n'en faisaient pas état. Pour remédier à cette situation, le projet a appliqué les pratiques de radio participative: d'abord, recueillir les savoirs locaux et les comprendre; ensuite, les mettre en relation avec le savoir des «experts».

Une première phase a consisté à écouter les ruraux, au cours de longues et nombreuses interviews semi-directives et de reportages dans les vil- lages. Ce matériel sonore a été analysé avec les partenaires de terrain (services techniques, ONG, etc.) dans un souci constant de comprendre le point de vue des ruraux. Les animateurs avaient reçu l'instruction de ne pas donner trop facilement raison aux experts, mais de bien faire apparaître le problème des ruraux et la manière de le surmonter. Par exemple, si des feux rituels sont vraiment indispensables pendant la période des feux tardifs, ils peuvent se faire à condition que des pare-feux soient préparés.

La campagne radiophonique est quant à elle plus directive, tout en donnant souvent la parole aux agriculteurs, éleveurs et chasseurs. Les messages directifs - «si vous avez quelque chose à brûler, c'est maintenant qu'il faut le faire», «la saison des feux précoces est terminée, on ne brûle plus » - sont construits à partir d'expressions fortes exprimées lors des interviews: il n'y a pas de meilleur expert qu'un paysan s'adressant à d'autres paysans.

La télédétection des feux joue un rôle central avant, pendant et après la campagne. La carte des feux a servi à déterminer les stations de radio à retenir pour la campagne. Les radios rurales dans sept pays de l'Afrique de l'Ouest (Bénin, Burkina, Guinée, Gambie, Mali, Niger et Sénégal) y ont finalement participé. Les cartes des feux mensuelles ont été communiquées aux communautés via le courrier électronique des radios rurales. Les ruraux se sentent ainsi observés du ciel, ce qui représente à leurs yeux un élément de motivation et même de valorisation de leur communauté, et non pas une intrusion de type Big Brother comme le craignaient des observateurs occidentaux. 
Le porte-parole d'un village guinéen près de Kankan aimait passionnément les feux. Loin de vouloir les combattre, il encourageait les villages voisins à les laisser passer et prendre de l'ampleur - quand bien même les villages sont parfois dévastés par les feux que leurs habitants ont eux-mêmes allumés.

Aux gens de la radio rurale qui lui parlaient des dangers de la désertification, il répondit: "Je reviens de La Mecque; là bas il n'y a plus d'arbres et pourtant les gens ne sont pas pauvres. " La radio rurale de Kankan a passé ce message en boucle, auquel elle a ajouté une seule question: "Etes-vous d'accord avec ce notable?»

Quelques jours plus tard le notable en question est venu supplier la radio d'arrêter cette diffusion. "Je me suis trompé", avouait-il piteusement. Tout le village avait réfléchi et s'était retourné contre lui.

Une évaluation de la campagne a montré des résultats très positifs.
Dans les zones d'écoute, les surfaces brûlées, tous feux confondus, ont diminué de 150’000 hectares, soit une régression de $40 \%$ environ ${ }^{2}$. Dans l'ensemble de l'Afrique de l'Ouest, les feux précoces ont augmenté, et les feux tardifs reculé.

Les TIC ne sont cependant pas toujours fiables. Les images satellites, qui devaient être distribuées chaque mois, ont mal circulé: le courrier électronique fonctionne irrégulièrement dans les régions rurales et des problèmes de format et d'accès à Internet freinent la circulation et l'exploitation des images. Il n'empêche qu'elles ont été communiquées, d'une manière ou d'une autre, presque partout et qu'elles ont été commentées par les radios. L'information a aussi circulé dans l'autre sens: certains villages ont confirmé au CSE que les incendies observés par satellite s'étaient bien produits, validation importante pour les techniciens basés à Dakar.

2 Les conditions climatiques ont certainement aussi joué un rôle dans ce résultat spectaculaire. 